\title{
High-density Grid Use for Left Lateral Accessory Pathway
}

\author{
CHELSEA PASKMAN, BS, ${ }^{1}$ ANDREA HAMMOND, BS, ${ }^{1}$ JOHN HARDING, $\mathrm{MD}^{2}{ }^{2}$ \\ and ROBERT SANGRIGOLI, MD ${ }^{2}$
}

\author{
${ }^{1}$ Abbott, Chicago, IL, USA \\ 2Doylestown Hospital, Doylestown, PA, USA
}

KEYWORDS. Advisor HD Grid, accessory pathway, AVRT, first burn termination, TactiCath sensor-enabled contact force ablation catheter.

\author{
ISSN 2156-3977 (print) \\ ISSN 2156-3993 (online) \\ CC BY 4.0 license \\ C 2021 Innovations in Cardiac \\ Rhythm Management
}

A 49-year-old woman with multiple documented episodes of narrow QRS complex tachycardia underwent electrophysiology study at our hospital. Baseline ventricular pacing demonstrated eccentric retrograde coronary sinus activation consistent with a left lateral accessory pathway (Figure 1A). Orthodromic tachycardia using a left lateral accessory pathway was repeatedly induced.

Transseptal puncture allowed access to the left atrium and left ventricle, where high-density mapping was performed across the mitral valve anulus during ventricular pacing using the Advisor ${ }^{\mathrm{TM}}$ HD Grid Mapping Catheter, Sensor Enabled ${ }^{\mathrm{TM}}$ and the EnSite Precision ${ }^{\mathrm{TM}}$ electroanatomic mapping system to localize the concealed left lateral accessory pathway. The open-window mapping technique ${ }^{1}$ was used by collecting the absolute $\mathrm{dV} / \mathrm{dt}$ bipolar electrogram from the high-density grid to help distinguish the mitral valve anulus, collecting both atrial and ventricular electrograms to delineate functional block located at the valve plane (Figure 1B). At the location of the accessory pathway, bipolar fusion and pathway potentials recognized and annotated on the high-density grid were able to showcase the atrial

Ms. Paskman and Ms. Hammond are employees of Abbott. The other authors report no conflicts of interest for the published content. Address correspondence to: Robert Sangrigoli, MD.

Email: rsangrigoli@dh.org. insertion point of the accessory pathway (Figures 1B and 1C). Additionally, during ventricular pacing and, similarly, during orthodromic supraventricular tachycardia, EnSite Precision ${ }^{\mathrm{TM}}$ open-window propagation and, in particular, the SparkleMap ${ }^{\mathrm{TM}}$ display feature (Figures 2A and 2B) allowed for more precise localization of the atrial insertion of the bypass tract by dynamically displaying wavefront propagation superimposed on top of the local activation timing and voltage maps (Videos 1 and 2).

Ablation was performed with the TactiCath ${ }^{\mathrm{TM}}$ Contact Force Ablation Catheter, Sensor Enabled ${ }^{\mathrm{TM}}$ at a power of $30 \mathrm{~W}$ and a minimal contact force of $10 \mathrm{~g}$ while the patient was in orthodromic supraventricular tachycardia. The tachycardia was terminated with a single lesion delivered to the mitral valve annulus at the atrial insertion site, subsequently eliminating the accessory pathway (Figure 1E). A thorough waiting period and electrophysiology study showed no evidence of accessory pathway conduction and the patient has remained symptom-free during follow-up.

\section{Reference}

1. Schricker AA, Winkle R, Moskovitz R, et al. Open-window mapping of accessory pathways utilizing high-density mapping. J Interv Card Electrophysiol. 2020 Aug 13. [Epub ahead of print]. 
A

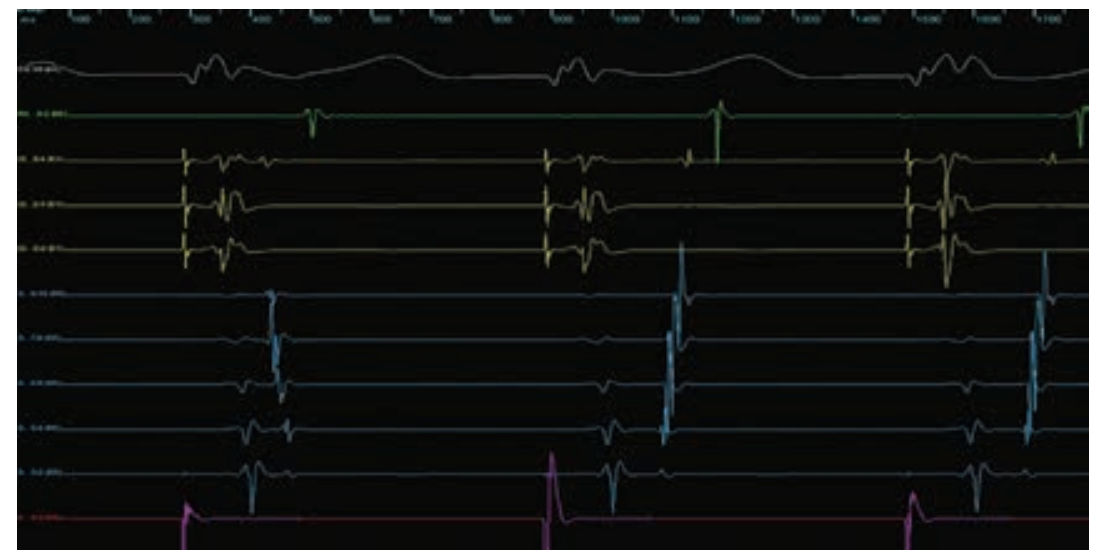

B

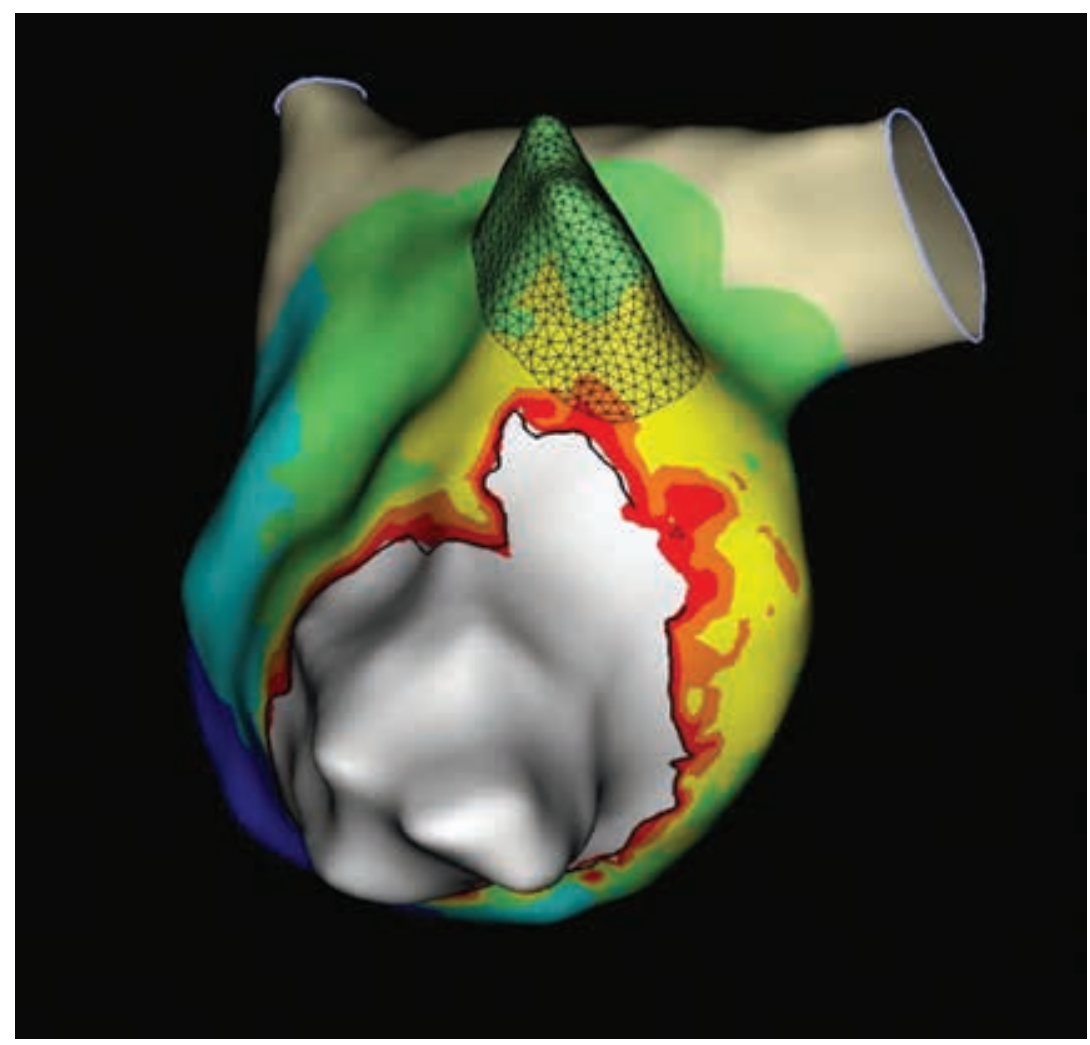

C

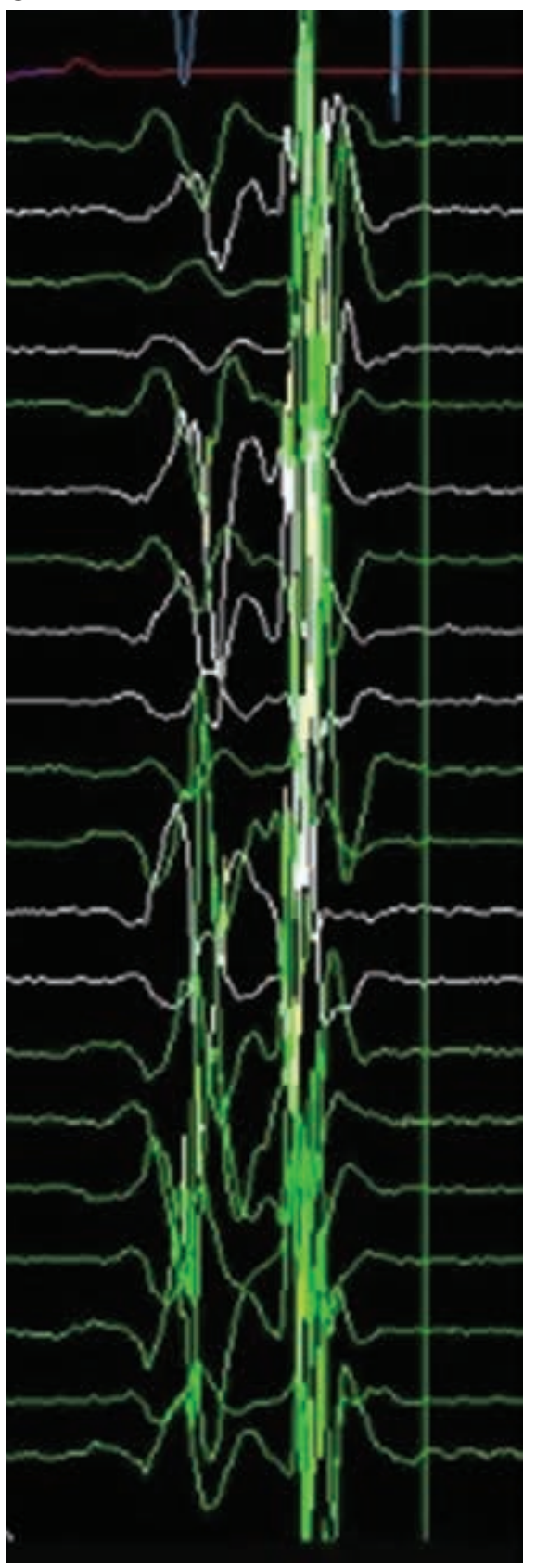

Figure 1: A: Ventricular pacing showcasing retrograde activation switching from atrioventricular nodal to accessory pathway conduction. B: EnSite Precision ${ }^{\mathrm{TM}}$ open window map of the left lateral accessory pathway using the Advisor ${ }^{\mathrm{TM}}$ HD Grid catheter. C: AdvisorTM HD Grid electrogram at the left lateral pathway location. 
D

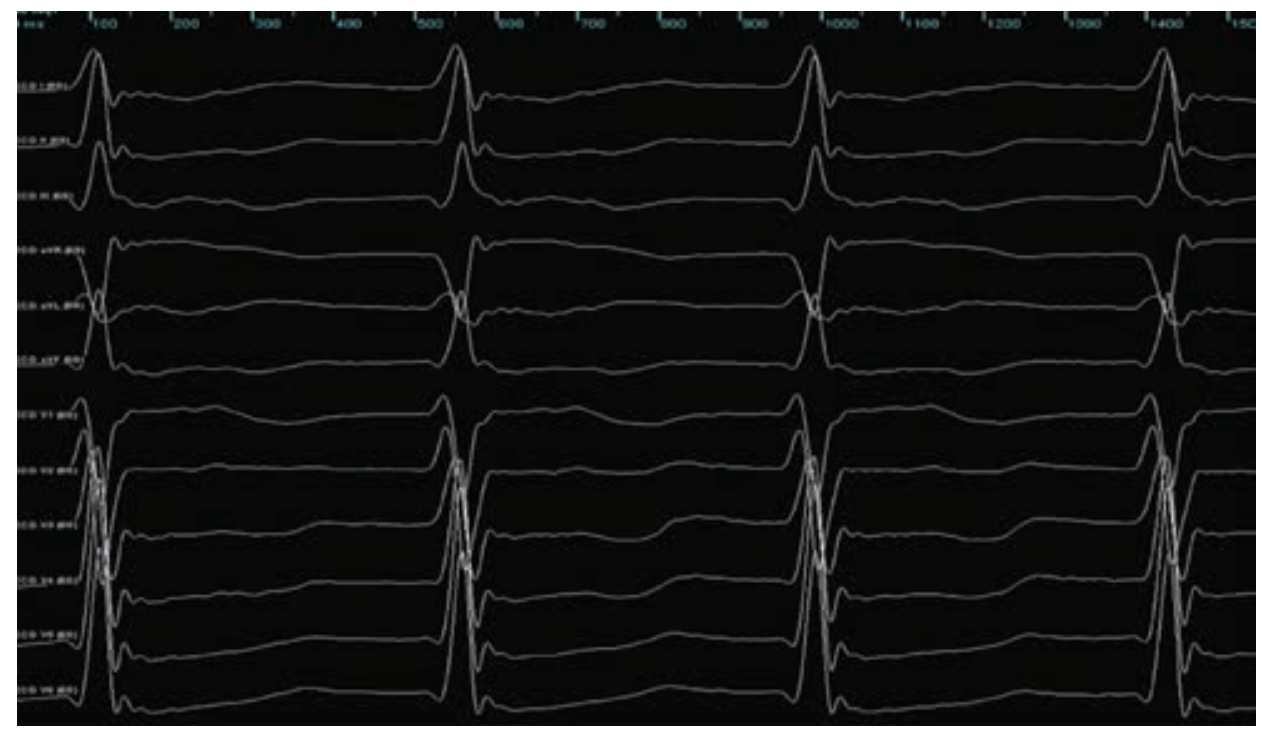

$E$

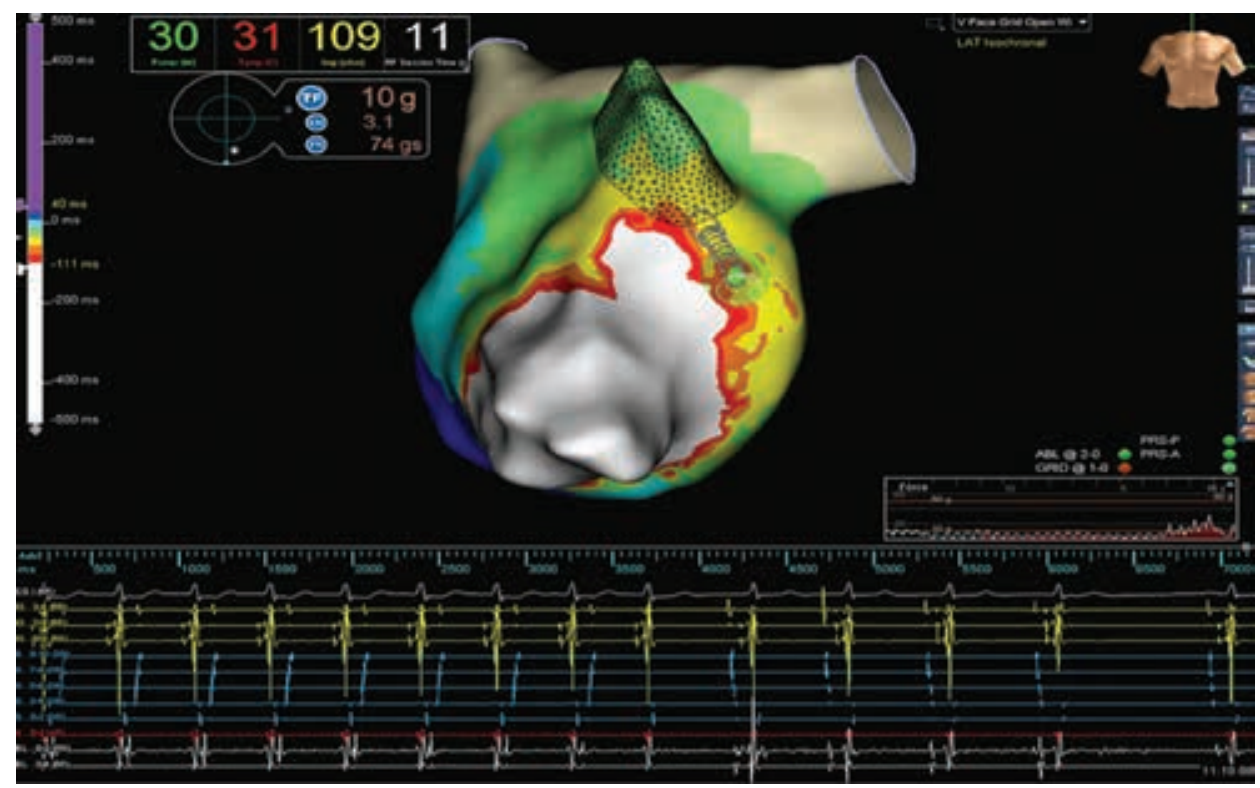

$\mathbf{F}$

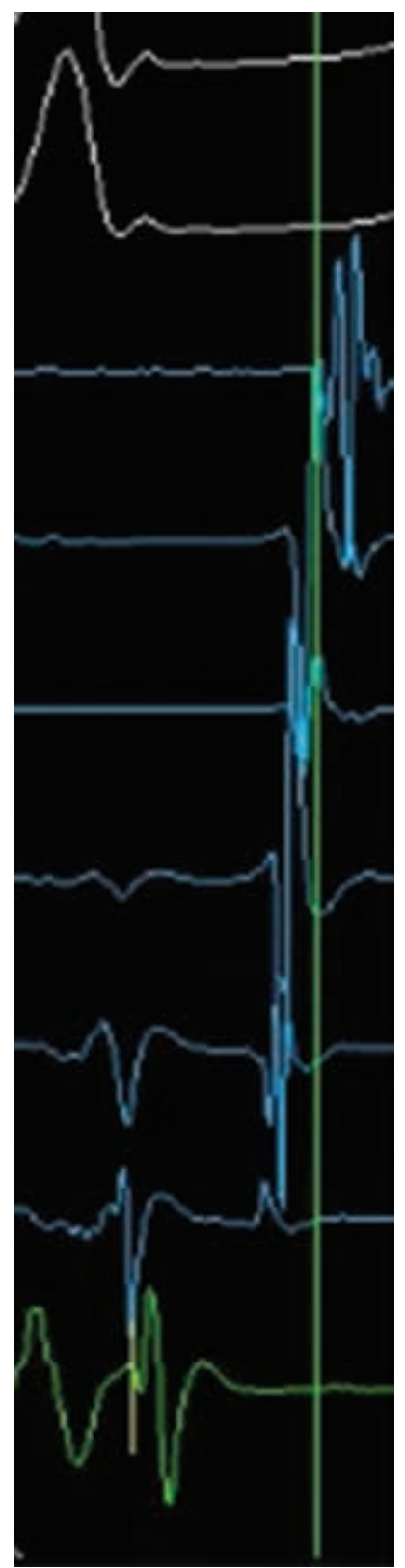

Figure 1: D: Clinical tachycardia 12-lead presentation. E: First burn termination during supraventricular tachycardia with the TactiCath $^{\mathrm{TM}}$ contact force catheter. F: Ventriculoatrial fusion at the site of successful ablation on the pathway. 


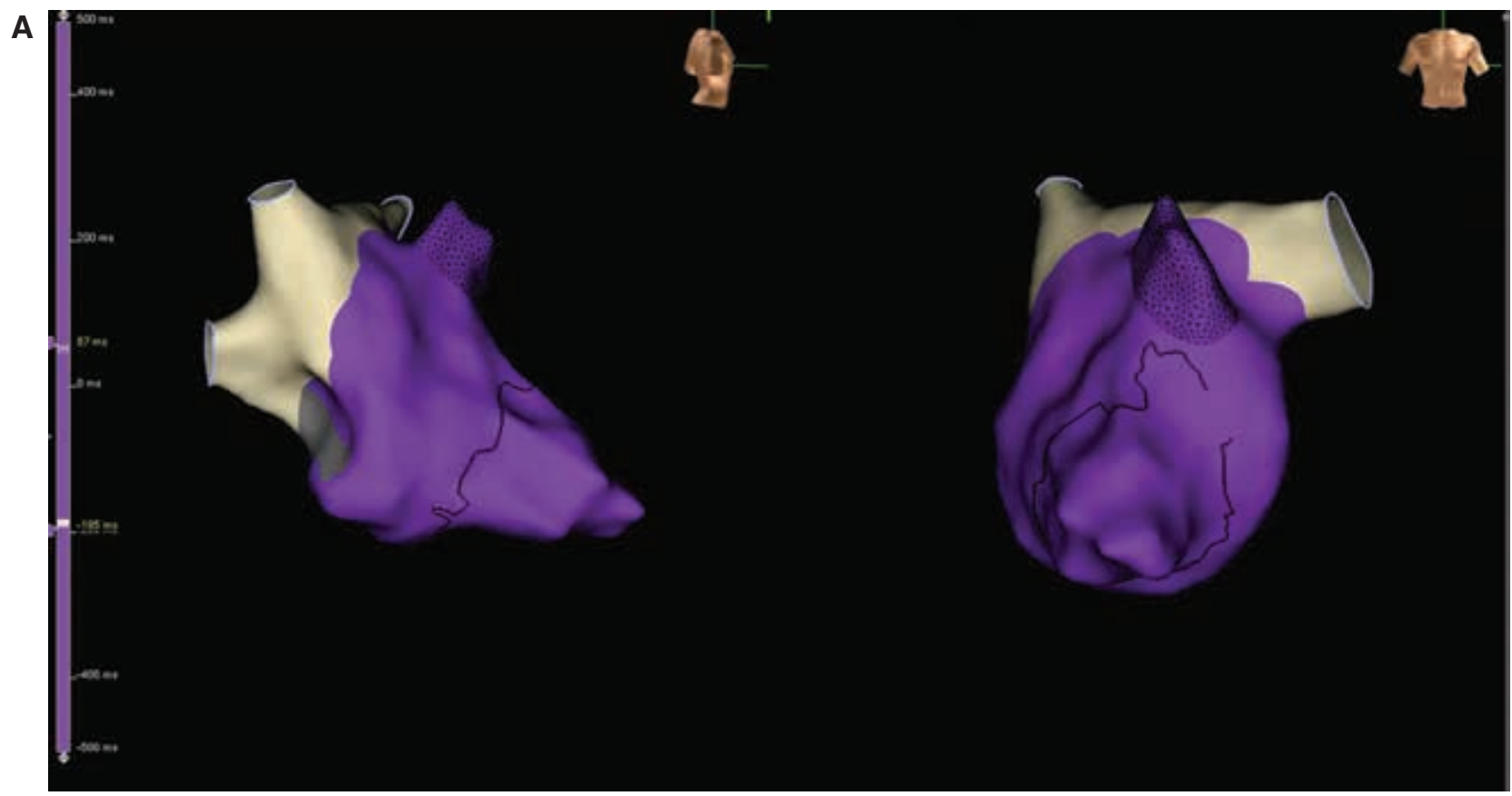

B

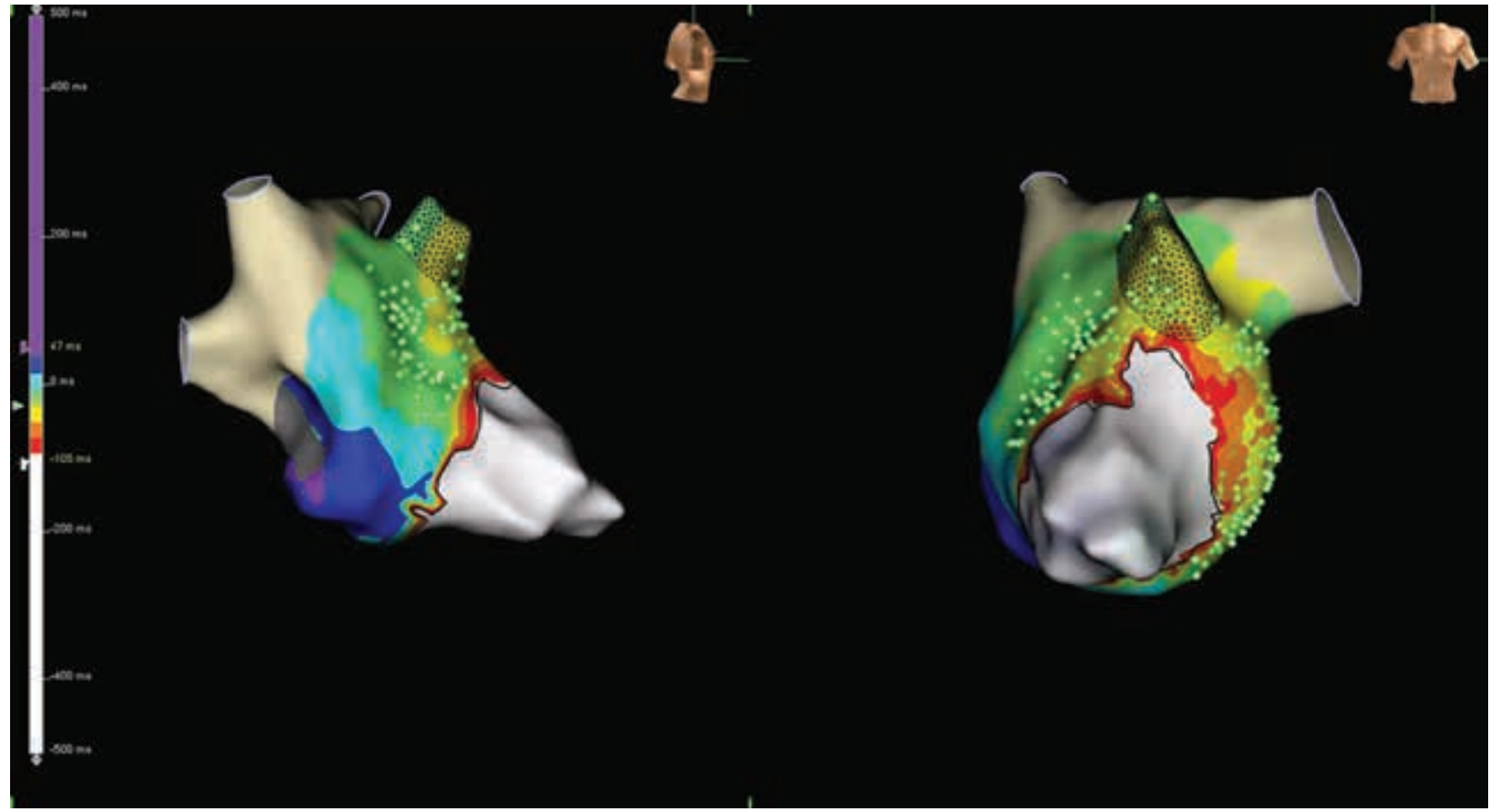

Figure 2: A: EnSite Precision ${ }^{\mathrm{TM}}$ open-window propagation video of the left lateral accessory pathway. B: EnSite Precision ${ }^{\mathrm{TM}}$ open-window map of the left lateral accessory pathway displayed with SparkleMap ${ }^{\mathrm{TM}}$. 